OPEN ACCESS

Edited by:

Junjie Xiao,

Shanghai University, China

Reviewed by:

Peng Zhang,

Capital Medical University, China

Li Li,

Ningbo First Hospital, China

*Correspondence:

Jianzhong $D$

dijianzhong@sjtu.com

Specialty section

This article was submitted to

Translational Endocrinology,

a section of the journal

Frontiers in Endocrinology

Received: 11 June 2020

Accepted: 28 July 2020

Published: 15 September 2020

Citation:

Xu T, Wang C, Zhang H, Han X, Liu W,

Han J, Yu H, Chen J, Zhang $P$ and Di J (2020) Timing of Maximal Weight

Reduction Following Bariatric Surgery:

A Study in Chinese Patients.

Front. Endocrinol. 11:615

doi: $10.3389 /$ fendo.2020.00615

\section{Timing of Maximal Weight Reduction Following Bariatric Surgery: A Study in Chinese Patients}

\author{
Ting $X{ }^{1}$, Chen Wang ${ }^{1}$, Hongwei Zhang ${ }^{1}$, Xiaodong Han ${ }^{1}$, Weijie Liu ${ }^{1}$, Junfeng Han ${ }^{2}$, \\ Haoyong $\mathrm{Yu}^{2}$, Jin Chen ${ }^{3}$, Pin Zhang ${ }^{1}$ and Jianzhong $\mathrm{Di}^{{ }^{*}}$
}

${ }^{1}$ Department of Bariatric \& Metabolic Surgery, Shanghai Jiao Tong University Affiliated Sixth People's Hospital, Shanghai, China, ${ }^{2}$ Department of Endocrinology and Metabolism, Shanghai Jiao Tong University Affiliated Sixth People's Hospital, Shanghai, China, ${ }^{3}$ Department of Computer Science, Institute for Biomedical Informatics, University of Kentucky, Lexington, KY, United States

Introduction: Bariatric surgery is a well-received treatment for obesity with maximal weight loss at 12-36 months postoperatively. We investigated the effect of early bariatric surgery on weight reduction of Chinese patients in accordance with their preoperation characteristics.

Materials and Methods: Altogether, 409 patients with obesity from a prospective cohort in a single bariatric center were enrolled retrospectively and evaluated for up to 4 years. Measurements obtained included surgery type, duration of diabetic condition, besides the usual body mass index data tuple. Weight reduction was expressed as percent total weight loss (\%TWL) and percent excess weight loss (\%EWL).

Results: RYGB or SG were performed laparoscopically without mortality or complications. BMl generally plateaued at 12 months, having decreased at a mean of $8.78 \mathrm{~kg} / \mathrm{m}^{2}$. Successful weight loss of $>25 \%$ TWL was achieved by $35.16,49.03$, $39.22,27.74,20.83 \%$ of patients at $6,12,24,36$, and 48 months after surgery. Overall, $52.91 \%$ of our patients had lost $100 \%$ of their excess weight at 12 months, although there was a rather wide range among individuals. Similar variability was revealed in women of child-bearing age.

Conclusion: Chinese patients undergoing bariatric surgery tend to achieve maximal weight loss and stabilization between 12 and 24 months postoperatively, instead of at $>2$ years. The finding of the shorter stabilization interval has importance to earlier intervention of weight loss related conditions and women's conception planning.

Keywords: bariatric surgery, Chinese patients, weight reduction, trend, follow up

\section{INTRODUCTION}

Obesity is a chronic health condition that is becoming a global issue. The World Health Organization stated in 2016 that more than 1.9 billion adults were overweight, and more than 650 million were obese. Besides lifestyle intervention and medication, bariatric surgery has been proved to be a safe, effective, and durable procedure for weight loss among morbidly obese patients (1). In addition, a number of comorbidities including essential hypertension, type 2 diabetes mellitus, hyperlipidemia, bronchial asthma, obstructive sleep apnea, and osteoarthritis can be ameliorated or 
even resolved following bariatric surgery. In China, the number of bariatric operations conducted has increased from around 4,000 as of the previous 5 year period to more than 10,000 during the past 5 years (2).

In western countries, research evidences support the consensus that postoperative body weight decreases to a trough at 12-36 months after bariatric surgery (3-5). However, there is currently no published investigation on standardized weight loss after bariatric surgery in Asian settings, except a few studies in Japan and Singapore showed that the average percent total weight loss (\%TWL) following bariatric surgery was $20-25 \%$ within the first 3 years $(6,7)$. On account of differences in the baseline body height and weight, and body composition, it is not completely grounded to interpret the weight loss on the Asian communities according to westerner physical standards.

The degree of weight loss and the time when it most likely occurs after bariatric surgery has been insufficiently addressed in China. In addition, we recognized in our patients that women who suffer from obesity-related infertility are more likely to have unplanned pregnancies within the first year following bariatric procedure. These altogether necessitate the establishment of the timing of maximal weight reduction during preoperative counseling. Patients who gain a realistic expectation of weight loss tend to stick to lifestyle changes and better cooperate with medical intervention, leading to uneventful recovery and satisfactory (8). To fulfill the need, we examined the up to 4 years' weight loss responses in Chinese patients following bariatric surgery according to their body mass index (BMI), age, sex, surgery type, and duration of type 2 diabetes mellitus (T2DM).

\section{METHODS}

We retrospectively reviewed the medical records of 409 patients who had undergone RYGB between February 2011 and August 2018. The Human Research Review Board of our institution approved the study and all patients provided their written informed consent. The study was conducted in accordance with the principles of the Declaration of Helsinki. Our previous studies focused on the improved renal, respiratory, and reproductive function after surgery (9-13). The present study includes bariatric surgery patients who underwent at least one follow-up evaluation during the first year after their surgery. Exclusion criteria included missing preoperative BMI record, unknown surgery type, death, and/or the presence of surgical complications.

Altogether, 409 patients were enrolled in the study (252 women, representing 61.6\%). Among them, 227 underwent Roux-en-Y gastric bypass (RYGB) (55.5\%), and the rest underwent sleeve gastrectomy (SG). Data were collected concerning the patients' demographic data, including their age and sex, T2DM diagnosis, type of surgery, initial BMI, and postoperative weight loss for up to 4 years (Table 1). Postoperative weight loss was expressed using the postoperative $\mathrm{BMI}$, change in BMI, \%TWL and \%EWL.
TABLE 1 | Baseline Characteristics.

\begin{tabular}{|c|c|}
\hline Parameter $(n=409)$ & Value \\
\hline $\mathrm{BMl}, \mathrm{kg} / \mathrm{m}^{2}$, Mean $\pm \mathrm{SD}$ (range) & $34.37 \pm 6.25(25.5,67.5)$ \\
\hline \multicolumn{2}{|l|}{ BMI group, $n(\%)$} \\
\hline $25-27.5 \mathrm{~kg} / \mathrm{m}^{2}$ & $22(5.4 \%)$ \\
\hline $27.5-32.5 \mathrm{~kg} / \mathrm{m}^{2}$ & 177 (43.3\%) \\
\hline $32.5-37.5 \mathrm{~kg} / \mathrm{m}^{2}$ & $104(25.4 \%)$ \\
\hline $37.5 \mathrm{~kg} / \mathrm{m}^{2}$ and above & $106(25.9 \%)$ \\
\hline Male sex, $n(\%)$ & $157(38.4 \%)$ \\
\hline \multicolumn{2}{|l|}{ T2DM duration, $n$ (\%) } \\
\hline Non-diabetic & $90(22.0 \%)$ \\
\hline $0-5$ years & $168(41.1 \%)$ \\
\hline $5-10$ years & $91(22.2 \%)$ \\
\hline $10-15$ years & 43 (10.5\%) \\
\hline above 15 years & $17(4.2 \%)$ \\
\hline Age, yrs, Mean \pm SD & $42.43 \pm 13.27$ \\
\hline \multicolumn{2}{|l|}{ Age, $n(\%)$} \\
\hline $18-45$ & 235 (57.5\%) \\
\hline $45-60$ & 118 (28.9\%) \\
\hline 60 and above & $56(13.7 \%)$ \\
\hline RYGB surgical type, $n(\%)$ & $227(55.5 \%)$ \\
\hline
\end{tabular}

BMI, body mass index; T2DM, type 2 diabetes mellitus; RYGB, Roux-en-Y gastric bypass.

\section{Statistical Analyses}

IBM SPSS Statistics software Version 20.0 (IBM Inc., Armonk, NY, USA) was used to analyze the data. Continuous data were analyzed for normality using the Shapiro-Wilks test. Differences between values were analyzed using the unpaired $t$ test (normal data), paired $t$-test (normal data), Mann-Whitney test (unpaired, non-normal data), or the Wilcoxon matched pairs test (non-normal data). A value of $p<0.05$ indicated statistical significance.

\section{RESULTS}

Detailed characteristics of our cohort are shown in Table 1 . There were more female than male patients ( 252 vs. 157). No patients had a BMI $<25 \mathrm{~kg} / \mathrm{m}^{2}$ (overweight), and there were 12 patients whose BMIs were $>50 \mathrm{~kg} / \mathrm{m}^{2}$ (super morbidly obese) at the time of their surgery. In all, 319 (78\%) patients were diagnosed with T2DM preoperatively. More patients (182 vs. 227) underwent RYGB than SG.

Postoperative changes in the BMI for up to 4 years are shown in Figure 1. The patients' BMI generally plateaued at 12 months, then decreased at a rate of $8.78 \mathrm{~kg} / \mathrm{m}^{2}$. Following a slight rebound, an insignificant downward trend was observed around the midterm, with the BMI decreasing to $\sim 25 \mathrm{~kg} / \mathrm{m}^{2}$. Weight loss of $>20 \%$ TWL was achieved by $75.62,64.66,57.42$, and $50.83 \%$ of patients at $12,24,36$, and 48 months after surgery. Up to $53.74 \%$ of our patients lost more than $100 \%$ of their excess weight (compared with a BMI of $25 \mathrm{~kg} / \mathrm{m}^{2}$ ) at 12 months. Although the mean weight loss stabilized at $\sim 24 \mathrm{~kg}$ at 12 months, there was wide variability among the individual values, especially between 


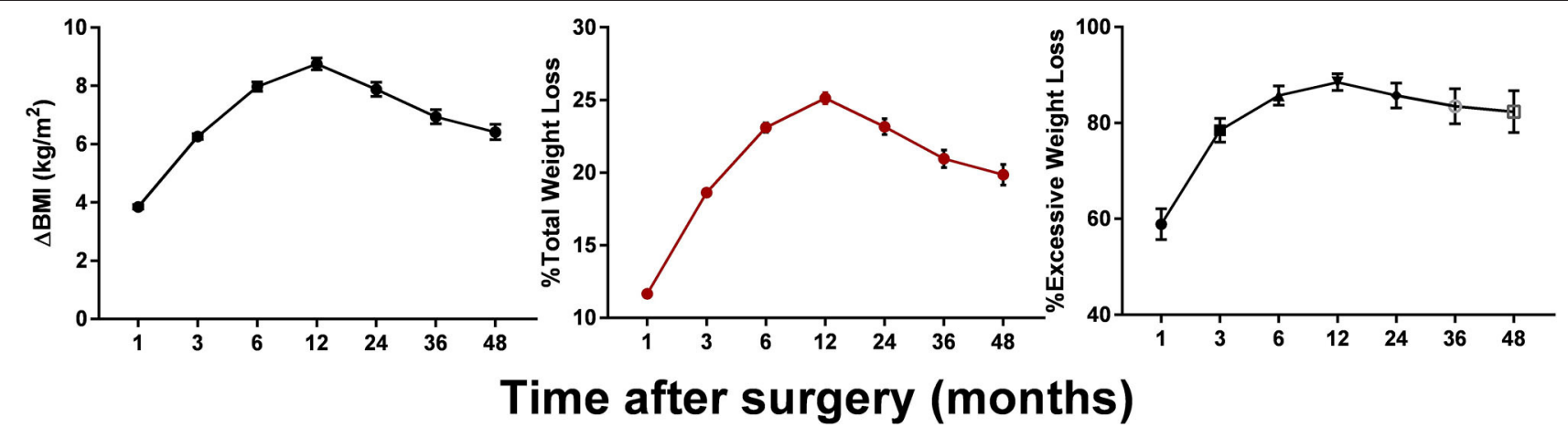

FIGURE 1 | Postoperative changes of $\triangle B M I, \% T W L$, and \%EWL. Results are shown in mean \pm SEM

the two surgical types. As can be seen from Table 2, weight loss after SG rebounded at 3 years after surgery, whereas after RYGB the weight loss was maintained.

Patients were also divided into groups based on certain characteristics to explore other possibilities (Figure 2). For example, there was no significant difference between the sexes, although the female patients had generally lost more weight than the male patients at the mid-term point. In addition, postRYGB weight loss followed a general pattern, whereas post-LSG weight loss fluctuated dramatically during the 3 years after the surgery. Divided into groups according to the preoperative BMI, patients with higher BMIs tended to lose more weight, although in a temperate slope, with the body weight dropping to its lowest 2 years postoperatively (vs. 1 year in other groups). Nondiabetic patients were more likely to have a significant weight rebound, and patients who had been diagnosed with T2DM for more than 15 years tended to face weight rebound as early as 6 months postoperatively.

The most common recipients of bariatric surgery were women of child-bearing age, accounting for $35.70 \%$ of our cohort (146 cases). Their mean BMI decreased from a baseline of 35.69 $\mathrm{kg} / \mathrm{m}^{2}$ to an ideal $25.23 \mathrm{~kg} / \mathrm{m}^{2}$ at 12 months and remaining at that level for 3 years postoperatively. Of these cases, we have also observed 17 pregnancies following bariatric surgery that result in different outcomes due to surgery-to-conception (S-C) interval. Two pregnancies were electively terminated during the first trimester for non-medical reasons. Five resulted in spontaneous abortions (defined as loss of pregnancy before 20 wk's gestation). The remaining 10 were carried to delivery.

\section{DISCUSSION}

This attempt to explore common bariatric weight loss criteria for Chinese patients showed that the body weight of bariatric surgery patients decreased sharply during the first 12-24 months, instead of at $>2$ years. It then stabilized unless the patient was nondiabetic, underwent SG, or had a high preoperative BMI, all of which make relapse more likely.
It is of interest to note that, the longer the patient's history of T2DM, the more likely it is that the body weight will decrease unsatisfactorily ( $84.7 \%$ for T2DM duration $>5$ years), if not rebound. This might be due to the different composition of body fat. For example, in a comparison with their BMImatched normoglycemic counterparts, patients with T2DM have increased hepatic and pancreatic fat, which causes a severe systemic inflammatory state. A conclusive explanation for this phenomenon, however, requires further study.

Comparison of the surgical effects of the gold standard RYGB vs. the relatively new, yet predominating, SG has become a topic of debate. Our data suggest that patients who undergo SG have a significantly higher BMI than those who undergo RYGB. Both groups plateaued at 12 months, but the SG group then relapsed by a mean $3.4 \mathrm{~kg} / \mathrm{m}^{2} 3$ years later. This conclusion differs from that derived in western countries, where plateaus usually start at 18 months, probably because of the higher baseline body weight and different dietary preferences. Longterm evidence of the effectiveness of SG remains sparse, although short- to medium-term results suggest that SG may not be inferior to RYGB (14). Our results may be biased because of a larger drop-off in the follow-up of our SG patients $(35.5 \%$ less than in the RYGB group). Also, because data were collected retrospectively in our study, some of the criteria selected were strongly related to whether SG was performed, including the duration of diabetes and baseline BMI. To justify the use of SG, randomized, controlled trials or cohort studies will be necessary to examine its benefit. In addition, a model that can better represent the effectiveness of bariatric surgery should be created, thereby neutralizing baseline differences that cannot be obviated.

Additionally, because most bariatric surgery is performed in women of reproductive age, defining the ideal interval between surgery and pregnancy is an emerging issue, which is partly based on the remission of obesity, which remains controversial in the present literature (15). Especially obese women in their late thirties who have failed many attempts to lose weight may look forward to a shorter interval for the good of both themselves and their children (16). Our research has indicated that women of reproductive age attain body weight stability at 12 months, which 
TABLE 2 | Reduction in BMl after surgery.

\begin{tabular}{|c|c|c|c|c|c|c|c|}
\hline & 1-m post-op & 3-m post-op & 6-m post-op & 1-y post-op & 2-y post-op & 3-y post-op & 4-y post-op \\
\hline$n$ & 242 & 322 & 347 & 361 & 232 & 155 & 120 \\
\hline Follow-up rate & $59 \%$ & $79 \%$ & $85 \%$ & $88 \%$ & $57 \%$ & $38 \%$ & $29 \%$ \\
\hline $\mathrm{BMl}, \mathrm{kg} / \mathrm{m}^{2}$ & $29.07 \pm 4.33$ & $27.20 \pm 4.40$ & $26.01 \pm 4.29$ & $25.28 \pm 3.88$ & $25.40 \pm 3.86^{\star}$ & $25.68 \pm 3.83^{\star}$ & $25.58 \pm 3.84^{*}$ \\
\hline RYGB & $27.84 \pm 3.18$ & $25.59 \pm 2.90$ & $24.50 \pm 3.03$ & $24.08 \pm 2.91$ & $24.66 \pm 3.34^{\star}$ & $25.16 \pm 3.34^{\star}$ & $25.26 \pm 3.64^{\star}$ \\
\hline SG & $33.30 \pm 5.04$ & $30.27 \pm 5.09$ & $28.26 \pm 4.87$ & $26.89 \pm 4.42$ & $27.38 \pm 4.54^{\star}$ & $28.46 \pm 5.04$ & $27.98 \pm 4.59^{*}$ \\
\hline$\Delta \mathrm{BMl}, \mathrm{kg} / \mathrm{m}^{2}$ & $3.85 \pm 1.22$ & $6.26 \pm 1.78$ & $7.97 \pm 2.89$ & $8.75 \pm 3.72$ & $7.88 \pm 3.66$ & $6.94 \pm 3.07$ & $6.42 \pm 2.85$ \\
\hline RYGB & $3.65 \pm 1.16$ & $5.77 \pm 1.52$ & $6.84 \pm 2.05$ & $7.18 \pm 2.24$ & $6.81 \pm 2.60$ & $6.34 \pm 2.45$ & $6.07 \pm 2.52$ \\
\hline SG & $4.53 \pm 1.21$ & $7.21 \pm 1.86$ & $9.67 \pm 3.14$ & $10.87 \pm 4.22$ & $10.75 \pm 4.47$ & $10.18 \pm 4.01$ & $8.98 \pm 3.79$ \\
\hline \%TWL & $11.65 \pm 3.11$ & $18.63 \pm 3.92$ & $23.11 \pm 5.88$ & $25.12 \pm 7.28$ & $23.16 \pm 8.13$ & $20.96 \pm 7.41$ & $19.86 \pm 7.76$ \\
\hline RYGB & $11.55 \pm 3.22$ & $18.32 \pm 4.10$ & $21.67 \pm 5.53$ & $22.81 \pm 6.15$ & $21.47 \pm 7.14$ & $20.01 \pm 7.40$ & $19.30 \pm 7.40$ \\
\hline SG & $12.00 \pm 2.71$ & $19.22 \pm 3.50$ & $25.27 \pm 5.75$ & $28.24 \pm 7.54$ & $27.72 \pm 8.90$ & $26.10 \pm 8.63$ & $24.02 \pm 9.29$ \\
\hline \%EWL & $58.88 \pm 25.04$ & $78.46 \pm 22.31$ & $85.71 \pm 18.60$ & $88.48 \pm 16.43$ & $85.72 \pm 20.03$ & $83.50 \pm 22.85$ & $82.33 \pm 24.02$ \\
\hline RYGB & $64.15 \pm 24.43$ & $85.59 \pm 18.57$ & $91.27 \pm 15.20$ & $92.23 \pm 14.28$ & $87.64 \pm 19.63$ & $85.06 \pm 22.34$ & $83.48 \pm 23.56$ \\
\hline SG & $40.83 \pm 17.79$ & $64.85 \pm 22.59$ & $77.31 \pm 20.11$ & $83.44 \pm 17.79$ & $80.55 \pm 20.35$ & $75.08 \pm 24.19$ & $73.81 \pm 26.56$ \\
\hline $\begin{array}{l}\text { Percentage of } \\
\text { follow-up patients } \\
\text { who reached } \\
\geq 50 \% E W L\end{array}$ & $11.98 \%$ & $38.20 \%$ & $49.86 \%$ & $52.91 \%$ & $51.29 \%$ & $52.90 \%$ & $50.83 \%$ \\
\hline $\begin{array}{l}\text { Percentage of } \\
\text { follow-up patients } \\
\text { who reached } \\
\geq 25 \% \text { TWL }\end{array}$ & $0.41 \%$ & $5.28 \%$ & $35.16 \%$ & $49.03 \%$ & $39.22 \%$ & $27.74 \%$ & $20.83 \%$ \\
\hline
\end{tabular}

$\% T W L=\frac{\text { Initial BMI-Final BMI }}{\text { Initial BMI }} \times 100 \%, \% E W L=\frac{\text { Initial BMI-Final BMI }}{\text { Initial BMI-Ideal BMI }} \times 100 \%,{ }^{*} P>0.05$ (vs. previous visit).

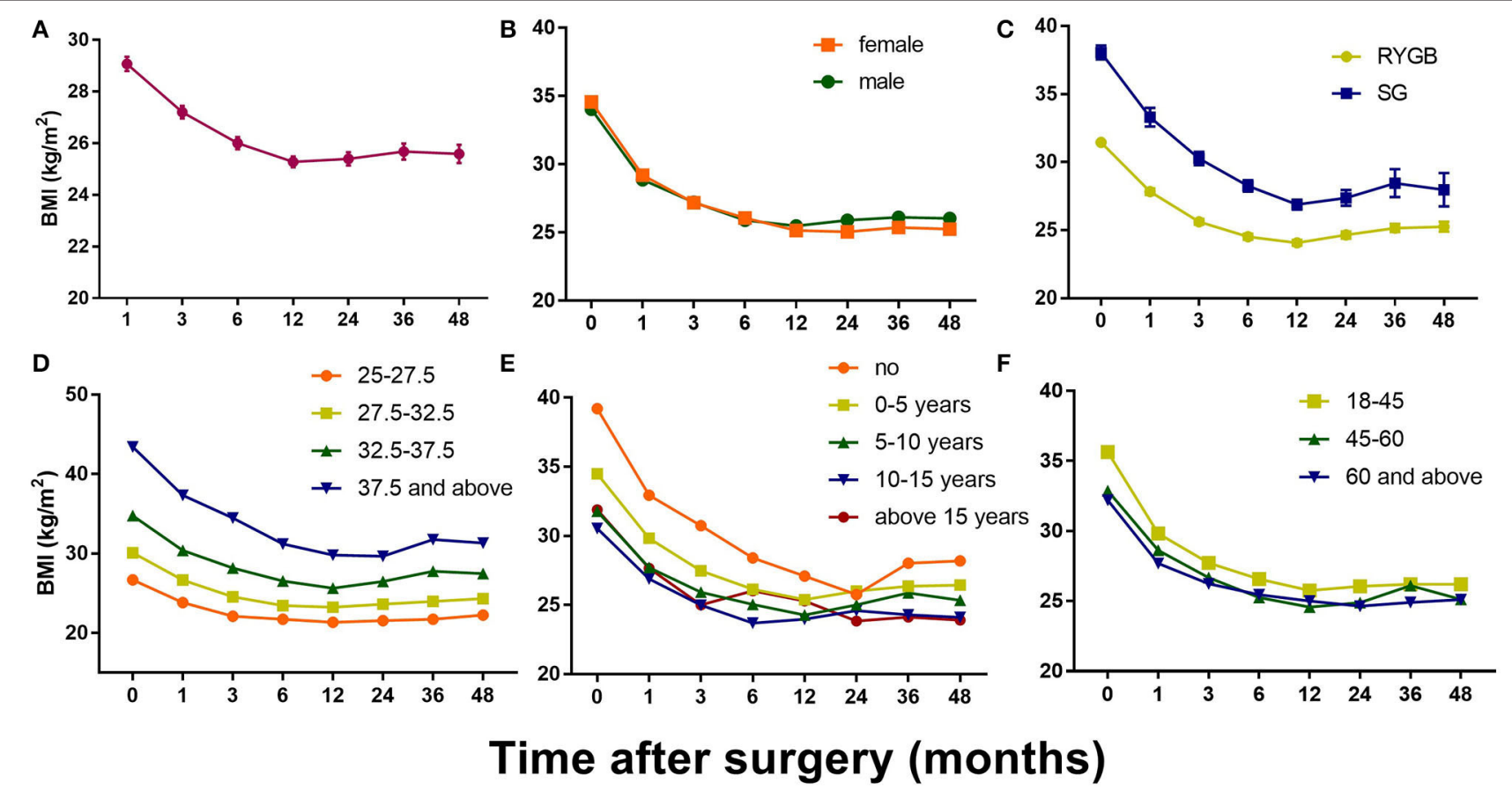

FIGURE 2 | Postoperative changes of BMI in all patients and in different groups. (A) Mean BMl change after bariatric surgery; (B) Mean BMI change in different gender groups; (C) Mean BMl change in different surgery type groups; (D) Mean BMl change in different initial BMl groups; (E) Mean BMl change in different T2DM duration groups; (F) Mean BMI change in different age groups. Results are shown in mean \pm SEM. 
indicates a mesomeric state suitable for pregnancy. Previous studies have shown that, in obese women with polycystic ovary syndrome, hormones such as estrogen and progestogen achieve a normalized balance as early as 3 months after bariatric surgery, which has led to unplanned pregnancies (17). Therefore, guidelines for bariatric surgery often suggest that pregnancy be avoided during the first $18-24$ months postoperatively-which is generally based on consensus instead of evidence (18). However, some studies have shown that there were almost no maternal or neonatal complications in women who became pregnant even within 6 months from the surgery $(18,19)$. In Asian settings, some research should be dedicated to determining whether the period between surgery and pregnancy can be shortened, and by how much. With our current cohort, the number of pregnancy cases was too small, thus more ought to be monitored in extended studies, in order to draw a rigorous conclusion over suggestions for conception.

The findings of this study have the potential to improve the perioperative care of patients undergoing bariatric surgery, highlighting areas for improvement in the follow-up protocol. As can be seen, although international standards (for RYGB) were followed regarding the follow-up routine, we could not identify a more precise "lowest body-weight period," which is vital to prevent regaining the lost weight. Therefore, more frequent monthly measurements between the 6 and 12 month follow-up evaluations can be scheduled and carried further beyond the first year. With this protocol, we hope to be able to identify patients who are at high risk for treatment failure at an early stage, so help can be provided promptly. Studies have reported the importance of developing good exercise and eating habits during the early postoperative period (20). Appropriate support can also be given to patients suffering from mental health issues such as depression or anxiety $(21,22)$.

There are some limitations of our study. First, as a retrospective trial, there is a risk of reporting and selection bias. To lessen the impact, data were collected prospectively, and all patients who met the inclusion criteria were enrolled consecutively in the study. Second, there was a large dropout rate, especially after 2 years postoperatively. More attention should be given to long-term follow-up, including the use of specialized personnel, standardized questionnaires, and professional databases. Third, the criteria we used to divide patients into groups were not sufficiently rigid (e.g., the surgery

\section{REFERENCES}

1. Puzziferri N, Roshek TB, Mayo HG, Gallagher R, Belle SH, Livingston EH. Long-term follow-up after bariatric surgery: a systematic review. JAMA. (2014) 312:934-42. doi: 10.1001/jama.2014.10706

2. Chinese Society for Metabolic \& Bariatric Surgery C. Guidelines on surgical treatment for obesity and type 2 diabetes mellitus in China. Chin J Pract Surg. (2019) 39:301-6. doi: 10.19538/j.cjps.issn1005-2208.2019.04.01

3. van de Laar AW, de Brauw M, Bruin SC, Acherman YI. Weight-independent percentile chart of 2880 gastric bypass patients: a new look at bariatric weight loss results. Obes Surg. (2016) 26:2891-98. doi: 10.1007/s11695-0162200-6 type may be dependent on diabetes duration and the BMI). The most essential criteria will be established in future studies.

\section{CONCLUSIONS}

Patients in a Chinese population achieved maximal weight loss and stabilization most probably between 12 and 24 months after surgery, instead of at $>2$ years. Further patient follow-up should include the time point of 18 month after surgery to establish more precise monthly time frames. The finding of the shorter stabilization interval is meaningful to earlier intervention of weight loss related conditions and women's conception planning.

\section{DATA AVAILABILITY STATEMENT}

The raw data supporting the conclusions of this article will be made available by the authors, without undue reservation.

\section{ETHICS STATEMENT}

The studies involving human participants were reviewed and approved by Shanghai Jiao Tong University affiliated Sixth People's Hospital. Written informed consent to participate in this study was provided by the participants' legal guardian/next of kin.

\section{AUTHOR CONTRIBUTIONS}

JD and PZ conceived the presented idea. TX developed the theory and performed the computations. CW and $\mathrm{HZ}$ verified the analytical methods. XH and WL performed the surgery. JH and HY helped with follow-ups. JC encouraged TX to investigate the impact of pregnancy on bariatric patients. JD supervised the findings of this work. All authors discussed the results and contributed to the final manuscript.

\section{FUNDING}

This study was supported by a clinical retrospective study of the Sixth People's Hospital Affiliated to Shanghai Jiaotong University, The middle-term efficacy of RYGB and SG in improving metabolic disorders (grant number: YNHG201912) and Tibet Autonomous Region Science and Technology Agency Project (grant number: XZ2017ZR-ZY014).
4. Homan J, Boerboom A, Aarts E, Dogan K, van Laarhoven C, Janssen I, et al. A longer biliopancreatic limb in Roux-en-Y gastric bypass improves weight loss in the first years after surgery: results of a randomized controlled trial. Obes Surg. (2018) 28:3744-55. doi: 10.1007/s11695-018-3421-7

5. Günther K, Vollmuth J, Weissbach R, Hohenberger W, Husemann B, Horbach T. Weight reduction after an early version of the open gastric bypass for morbid obesity: results after 23 years. Obes Surg. (2006) 16:28896. doi: 10.1381/096089206776116543

6. Haruta H, Kasama K, Ohta M, Sasaki A, Yamamoto H, Miyazaki $\mathrm{Y}$, et al. Long-term outcomes of bariatric and metabolic surgery in Japan: results of a multi-institutional survey. Obes Surg. (2017) 27:75462. doi: 10.1007/s11695-016-2361-3 
7. Toh BC, Chan WH, Eng AKH, Lim EKW, Lim CH, Tham KW, et al. Fiveyear long-term clinical outcome after bariatric metabolic surgery: a multiethnic Asian population in Singapore. Diabetes Obes Metab. (2018) 20:17625. doi: 10.1111/dom.13263

8. Aelfers SCW, Schijns W, Ploeger N, Janssen IMC, Berends FJ, Aarts EO. Patients' preoperative estimate of target weight and actual outcome after bariatric surgery. Obes Surg. (2017) 27:1729-34. doi: 10.1007/s11695-017-2556-2

9. Zhang $\mathrm{H}$, Di J, Yu H, Han $\mathrm{X}$, Li K, Zhang P. The short-term remission of diabetic nephropathy after Roux-en-Y gastric bypass in Chinese patients of T2DM with obesity. Obes Surg. (2015) 25:126370. doi: 10.1007/s11695-015-1666-y

10. Zhang H, Han X, Yu H, Di J, Zhang P, Jia W. Effect of Roux-en-Y gastric bypass on remission of T2D: medium-term follow-up in Chinese patients with different BMI obesity class. Obes Surg. (2017) 27:13442. doi: 10.1007/s11695-016-2262-5

11. Zou J, Zhang P, Yu H, Di J, Han X, Yin S, et al. Effect of laparoscopic Roux-en-Y gastric bypass surgery on obstructive sleep apnea in a Chinese population with obesity and T2DM. Obes Surg. (2015) 25:144653. doi: 10.1007/s11695-014-1510-9

12. Kun L, Pin Z, Jianzhong D, Xiaodong H, Haoyong Y, Yuqian $B$, et al. Significant improvement of erectile function after Rouxen-Y gastric bypass surgery in obese Chinese men with erectile dysfunction. Obes Surg. (2015) 25:838-44. doi: 10.1007/s11695-0141465 -x

13. Li K, Zou J, Ye Z, Di J, Han X, Zhang H, et al. Effects of bariatric surgery on renal function in obese patients: a systematic review and meta analysis. PLoS ONE. (2016) 11:e0163907. doi: 10.1371/journal.pone.01 63907

14. Golzarand M, Toolabi K, Farid R. The bariatric surgery and weight losing: a meta-analysis in the long- and very long-term effects of laparoscopic adjustable gastric banding, laparoscopic Roux-en-Y gastric bypass and laparoscopic sleeve gastrectomy on weight loss in adults. Surg Endosc. (2017) 31:4331-45. doi: 10.1007/s00464-017-5505-1

15. Johansson K, Cnattingius S, Näslund I, Roos N, Lagerros YT, Granath F, et al. Outcomes of pregnancy after bariatric surgery. N Engl J Med. (2015) 372:814-24. doi: 10.1056/NEJMoa1405789
16. Cooney LG, Dokras A. Depression and anxiety in polycystic ovary syndrome: etiology and treatment. Curr Psychiatry Rep. (2017) 19:83. doi: $10.1007 /$ s11920-017-0834-2

17. Christ JP, Falcone T. Bariatric surgery improves hyperandrogenism, menstrual irregularities, and metabolic dysfunction among women with polycystic ovary syndrome (PCOS). Obes Surg. (2018) 28:2171-7. doi: 10.1007/s11695-018-3155-6

18. Conway G, Dewailly D, Diamanti-Kandarakis E, Escobar-Morreale HF, Franks S, Gambineri A, et al. The polycystic ovary syndrome: a position statement from the European Society of Endocrinology. Eur. J. Endocrinol. (2014) 171:P1-29. doi: 10.1530/EJE-14-0253

19. Yau PO, Parikh M, Saunders JK, Chui P, Zablocki T, Welcome AU. Pregnancy after bariatric surgery: the effect of time-toconception on pregnancy outcomes. Surg Obes Relat Dis. (2017) 13:1899-905. doi: 10.1016/j.soard.2017.07.015

20. Froylich D, Corcelles R, Daigle CR, Kirwan JP, Brethauer SA, Schauer PR, et al. The effect of pregnancy before and/or after bariatric surgery on weight loss. Surg Obes Relat Dis. (2016) 12:596-599. doi: 10.1016/j.soard.2015.09.005

21. Amin MN, Hussain MS, Sarwar MS, Rahman Moghal MM, Das A, Hossain $\mathrm{MZ}$, et al. How the association between obesity and inflammation may lead to insulin resistance and cancer. Diabetes Metab Syndr. (2019) 13:121324. doi: 10.1016/j.dsx.2019.01.041

22. Coulman KD, MacKichan F, Blazeby JM, Owen-Smith A. Patient experiences of outcomes of bariatric surgery: a systematic review and qualitative synthesis. Obes Rev. (2017) 18:547-59. doi: 10.1111/obr.12518

Conflict of Interest: The authors declare that the research was conducted in the absence of any commercial or financial relationships that could be construed as a potential conflict of interest.

Copyright (๑) $2020 \mathrm{Xu}$, Wang, Zhang, Han, Liu, Han, Yu, Chen, Zhang and Di. This is an open-access article distributed under the terms of the Creative Commons Attribution License (CC BY). The use, distribution or reproduction in other forums is permitted, provided the original author(s) and the copyright owner(s) are credited and that the original publication in this journal is cited, in accordance with accepted academic practice. No use, distribution or reproduction is permitted which does not comply with these terms. 